ISSN 2075-9827 e-ISSN 2313-0210

Carpathian Math. Publ. 2020, 12 (1), 5-16

doi:10.15330/cmp.12.1.5-16

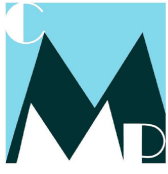

http://www.journals.pnu.edu.ua/index.php/cmp

Карпатські матем. публ. 2020, Т.12, №1, C.5-16

\title{
VASYLYSHYN T.V.
}

\section{SYMMETRIC FUNCTIONS ON SPACES $\ell_{p}\left(\mathbb{R}^{n}\right)$ AND $\ell_{p}\left(\mathbb{C}^{n}\right)$}

This work is devoted to the study of algebras of continuous symmetric polynomials, that is, invariant with respect to permutations of coordinates of its argument, and of $*$-polynomials on Banach spaces $\ell_{p}\left(\mathbb{R}^{n}\right)$ and $\ell_{p}\left(\mathbb{C}^{n}\right)$ of $p$-power summable sequences of $n$-dimensional vectors of real and complex numbers respectively, where $1 \leq p<+\infty$.

We construct the subset of the algebra of all continuous symmetric polynomials on the space $\ell_{p}\left(\mathbb{R}^{n}\right)$ such that every continuous symmetric polynomial on the space $\ell_{p}\left(\mathbb{R}^{n}\right)$ can be uniquely represented as a linear combination of products of elements of this set. In other words, we construct an algebraic basis of the algebra of all continuous symmetric polynomials on the space $\ell_{p}\left(\mathbb{R}^{n}\right)$. Using this result, we construct an algebraic basis of the algebra of all continuous symmetric $*$-polynomials on the space $\ell_{p}\left(\mathbb{C}^{n}\right)$.

Results of the paper can be used for investigations of algebras, generated by continuous symmetric polynomials on the space $\ell_{p}\left(\mathbb{R}^{n}\right)$, and algebras, generated by continuous symmetric $*$-polynomials on the space $\ell_{p}\left(\mathbb{C}^{n}\right)$.

Key words and phrases: polynomial, $*$-polynomial, symmetric polynomial, symmetric $*$-polynomial, algebraic basis.

Vasyl Stefanyk Precarpathian National University, 57 Shevchenka str., 76018, Ivano-Frankivsk, Ukraine

E-mail: taras.v.vasylyshynegmail.com

\section{INTRODUCTION}

Symmetric (invariant with respect to some group of operators) functions on Banach spaces were studied by a number of authors $[1-8,10,11,15-21,23]$. In particular, in [15] it was constructed an algebraic basis (see definition below) of the algebra of all continuous symmetric, i.e., invariant with respect to permutations of coordinates of its argument, polynomials on the real Banach space $\ell_{p}$ of $p$-power summable sequences of real numbers, where $1 \leq p<+\infty$. In [8] it was generalized this result to continuous symmetric polynomials on real separable rearrangement-invariant sequence Banach spaces. In [11] it was constructed an algebraic basis of the algebra of all continuous symmetric polynomials on the complex Banach space $\ell_{p}\left(\mathbb{C}^{n}\right)$ of $p$-power summable sequences of $n$-dimensional vectors of complex numbers, where $1 \leq p<+\infty$. Note that the knowledge of an algebraic basis of an algebra of polynomials is important for the description of spectra (sets of maximal ideals) of completions of this algebra (see, e.g., $[1,4,5,7,9,17]$ ).

*-Polynomials (see definition below) are generalizations of polynomials, acting between complex vector spaces, which were firstly studied in [14]. In [13] it was shown that, in some sense, *-polynomials have better approximation properties than polynomials. Symmetric $*-$ polynomials on the finite-dimensional complex vector space were studied in [16]. In particular, 
in [16] it was constructed the set of generating elements of the algebra of all symmetric *polynomials on the complex space of finite sequences of $n$-dimensional complex vectors.

In this work we construct an algebraic basis of the algebra of all continuous symmetric polynomials on the real Banach space $\ell_{p}\left(\mathbb{R}^{n}\right)$ of $p$-power summable sequences of $n$-dimensional vectors of real numbers, where $1 \leq p<+\infty$. Also we construct an algebraic basis of the algebra of all continuous symmetric $*$-polynomials on the complex Banach space $\ell_{p}\left(\mathbb{C}^{n}\right)$.

\section{PRELIMINARIES}

Let $\mathbb{N}$ be the set of all positive integers and $\mathbb{Z}_{+}$be the set of all nonnegative integers. Let $\mathcal{S}$ be the set of all bijections $\sigma: \mathbb{N} \rightarrow \mathbb{N}$. For $n \in \mathbb{N}$, let $\mathcal{S}_{n}$ be the set of all bijections $\sigma:\{1, \ldots, n\} \rightarrow\{1, \ldots, n\}$.

\subsection{Polynomials}

Let $X$ and $Y$ be vector spaces over the fields $\mathbb{K}_{1}$ and $\mathbb{K}_{2}$ resp., such that $\mathbb{K}_{1} \subset \mathbb{K}_{2}$ and $\mathbb{K}_{1}, \mathbb{K}_{2} \in\{\mathbb{R}, \mathbb{C}\}$. A mapping $A: X^{m} \rightarrow Y$, where $m \in \mathbb{N}$, is called an m-linear mapping, if $A$ is linear with respect to every of its $m$ arguments. An $m$-linear mapping, which is invariant with respect to permutations of its arguments is called symmetric. For an $m$-linear mapping $A: X^{m} \rightarrow Y$, let $A^{(s)}: X^{m} \rightarrow Y$ be defined by

$$
A^{(s)}\left(x_{1}, \ldots, x_{m}\right)=\frac{1}{m !} \sum_{\tau \in \mathcal{S}_{m}} A\left(x_{\tau(1)}, \ldots, x_{\tau(m)}\right) .
$$

The mapping $A^{(s)}$ is symmetric and $m$-linear. It is called the symmetrization of the mapping $A$. A mapping $P: X \rightarrow Y$ is called an $m$-homogeneous polynomial if there exists an $m$-linear mapping $A_{P}: X^{m} \rightarrow Y$ such that $P$ is the restriction to the diagonal of $A_{P}$, i.e., $P(x)=A_{P}(\underbrace{x, \ldots, x}_{m})$ for every $x \in X$. Note that $P$ is also the restriction to the diagonal of the mapping $A_{P}^{(s)}$, which is the symmetrization of the mapping $A_{P}$. The mapping $A_{P}^{(s)}$ is called the symmetric $m$-linear mapping, associated with $P$. By [14, Theorem 1.10, p. 6], the mapping $A_{P}^{(s)}$ can be recovered by the values of $P$ by means of the formula

$$
A_{P}^{(s)}\left(x_{1}, \ldots, x_{m}\right)=\frac{1}{2^{m} m !} \sum_{\varepsilon_{1}, \ldots, \varepsilon_{m}= \pm 1} \varepsilon_{1} \ldots \varepsilon_{m} P\left(\varepsilon_{1} x_{1}+\ldots+\varepsilon_{m} x_{m}\right) .
$$

For convenience, we define 0 -homogeneous polynomials from $X$ to $Y$ as constant mappings. A mapping $P: X \rightarrow Y$ is called a polynomial if it can be represented in the form

$$
P=\sum_{j=0}^{K} P_{j},
$$

where $K \in \mathbb{Z}_{+}$and $P_{j}$ is a $j$-homogeneous polynomial for every $j \in\{0, \ldots, K\}$. Let deg $P$ be the maximal number $j \in \mathbb{Z}_{+}$, such that $P_{j} \not \equiv 0$.

For, in general, complex numbers $t_{1}, \ldots, t_{m}$, let $V_{t_{1}, \ldots, t_{m}}$ be the Vandermonde matrix

$$
V_{t_{1}, \ldots, t_{m}}:=\left(\begin{array}{ccccc}
1 & t_{1} & t_{1}^{2} & \ldots & t_{1}^{m-1} \\
1 & t_{2} & t_{2}^{2} & \ldots & t_{2}^{m-1} \\
\vdots & \vdots & \vdots & \ddots & \vdots \\
1 & t_{m} & t_{m}^{2} & \ldots & t_{m}^{m-1}
\end{array}\right) .
$$


The following proposition gives us the method of recovering of homogeneous components of any polynomial $P$ by its values.

Proposition 1 ([12]). Let $P$ be a polynomial of the form (2). Let $\lambda_{0}, \ldots, \lambda_{K}$ be distinct nonzero real numbers. Then

$$
P_{j}(x)=\sum_{s=0}^{K} w_{j s} P\left(\lambda_{s} x\right)
$$

for every $j \in\{0, \ldots, K\}$, where $w_{j s}$ are elements of the matrix $W=\left(w_{j s}\right)_{j, s=\overline{0, K}}$, which is the inverse matrix of the Vandermonde matrix $V_{\lambda_{0}, \ldots, \lambda_{K}}$.

Suppose $X$ and $Y$ are normed spaces with norms $\|\cdot\|_{X}$ and $\|\cdot\|_{Y}$ respectively. Note that an $m$-linear mapping $A: X^{m} \rightarrow Y$ is continuous if and only if the value

$$
\|A\|=\sup _{\left\|x_{1}\right\|_{X} \leq 1, \ldots,\left\|x_{m}\right\|_{X} \leq 1}\left\|A\left(x_{1}, \ldots, x_{m}\right)\right\|_{Y}
$$

is finite. Similarly, an $m$-homogeneous polynomial $P: X \rightarrow Y$ is continuous if and only if the value

$$
\|P\|=\sup _{\|x\|_{X} \leq 1}\|P(x)\|_{Y}
$$

is finite. By definitions of $\|P\|$ and $\left\|A_{P}\right\|$, and by formula (1),

$$
\|P\| \leq\left\|A_{P}^{(s)}\right\| \leq \frac{m^{m}}{m !}\|P\| \text {. }
$$

\section{$1.2 *$ *-Polynomials}

Let $X$ and $Y$ be complex vector spaces. A mapping $A: X^{m+n} \rightarrow Y$, where $(m, n) \in$ $\mathbb{Z}_{+}^{2} \backslash\{(0,0)\}$, is called an $(m, n)$-linear mapping, if $A$ is linear with respect to every of first $m$ arguments and it is antilinear with respect to every of last $n$ arguments. An $(m, n)$-linear mapping, which is invariant with respect to permutations of its first $m$ arguments and last $n$ arguments separately, is called $(m, n)$-symmetric. For $(m, n)$-linear mapping $A: X^{m+n} \rightarrow Y$, let $A^{(s)}: X^{m+n} \rightarrow Y$ be defined by

$$
A^{(s)}\left(x_{1}, \ldots, x_{m}, x_{m+1}, \ldots, x_{m+n}\right)=\frac{1}{m ! n !} \sum_{\tau \in \mathcal{S}_{m}} \sum_{\theta \in \mathcal{S}_{n}} A\left(x_{\tau(1)}, \ldots, x_{\tau(m)}, x_{m+\theta(1)}, \ldots, x_{m+\theta(n)}\right) .
$$

The mapping $A^{(s)}$ is $(m, n)$-symmetric and $(m, n)$-linear. It is called the $(m, n)$-symmetrization of the mapping $A$. A mapping $P: X \rightarrow Y$ is called an $(m, n)$-polynomial if there exists an $(m, n)$-linear mapping $A_{P}: X^{m+n} \rightarrow Y$ such that $P$ is the restriction to the diagonal of $A_{P}$, i.e.,

$$
P(x)=A_{P}(\underbrace{x, \ldots, x}_{m+n})
$$

for every $x \in X$. Note that $P$ is also the restriction to the diagonal of the mapping $A_{P}^{(s)}$, which is the $(m, n)$-symmetrization of the mapping $A_{P}$. The mapping $A_{P}^{(s)}$ is called the $(m, n)$ symmetric $(m, n)$-linear mapping, associated with $P$. By [22, Theorem 3.1], the mapping $A_{P}^{(s)}$ can be recovered by the values of $P$ by means of the formula

$$
\begin{aligned}
A_{P}^{(s)}\left(x_{1}, \ldots, x_{m+n}\right) & =\frac{1}{2^{m+n} m ! n !} \sum_{\varepsilon_{1}, \ldots, \varepsilon_{m+n}= \pm 1} \varepsilon_{1} \ldots \varepsilon_{m+n} \sum_{j=1}^{2 n+1} \frac{1}{2 n+1} \alpha_{j}^{2 n+1-m} \\
& \times P\left(\alpha_{j}\left(\varepsilon_{1} x_{1}+\ldots+\varepsilon_{m} x_{m}\right)+\varepsilon_{m+1} x_{m+1}+\ldots+\varepsilon_{m+n} x_{m+n}\right),
\end{aligned}
$$


where $\alpha_{j}=e^{2 \pi i j /(2 n+1)}$ for $j \in\{1, \ldots, 2 n+1\}$.

For convenience, we define $(0,0)$-polynomials from $X$ to $Y$ as constant mappings.

A mapping $P: X \rightarrow Y$ is called a $*$-polynomial if it can be represented in the form

$$
P=\sum_{t=0}^{K} \sum_{j=0}^{t} P_{j, t-j},
$$

where $K \in \mathbb{Z}_{+}$and $P_{j, t-j}$ is a $(j, t-j)$-polynomial for every $t \in\{0, \ldots, K\}$ and $j \in\{0, \ldots, t\}$. Let $\operatorname{deg} P$ be the maximal number $t \in \mathbb{Z}_{+}$, for which there exists $j \in\{0, \ldots, t\}$ such that $P_{j, t-j} \not \equiv 0$.

Results from [16, Proposition 1] and [16, Proposition 2] imply the following proposition, which gives us the method of recovering of components of any $*$-polynomial $P$ by its values.

Proposition 2. Let $P: X \rightarrow Y$ be a *-polynomial of the form (5), where $X$ and $Y$ are complex vector spaces. Let $\lambda_{0}, \ldots, \lambda_{K}$ be distinct nonzero real numbers. Let $\varepsilon_{0}, \ldots, \varepsilon_{K}$ be complex numbers such that $\varepsilon_{0}^{2}, \ldots, \varepsilon_{K}^{2}$ are distinct and $\left|\varepsilon_{0}\right|=\ldots=\left|\varepsilon_{K}\right|=1$. Then

$$
P_{j, t-j}(x)=\sum_{l=0}^{t} u_{j l} \varepsilon_{l}^{t} \sum_{s=0}^{K} w_{t s} P\left(\lambda_{s} \varepsilon_{l} x\right)
$$

for every $t \in\{0, \ldots, K\}, j \in\{0, \ldots, t\}$ and $x \in X$, where $w_{t s}$ are elements of the matrix $W=\left(w_{t s}\right)_{t, s=\overline{0, K},}$, which is the inverse matrix of the Vandermonde matrix $V_{\lambda_{0}, \ldots, \lambda_{K}}$, and $u_{j l}$ are elements of the matrix $U=\left(u_{j l}\right)_{j, l}=\overline{0, K}$, which is the inverse matrix of the Vandermonde matrix $V_{\varepsilon_{0}^{2}, \ldots, \varepsilon_{K}^{2}}$.

Suppose $X$ and $Y$ are complex normed spaces with norms $\|\cdot\|_{X}$ and $\|\cdot\|_{Y}$ resp. Note that an $(m, n)$-linear mapping $A: X^{m+n} \rightarrow Y$ is continuous if and only if the value

$$
\|A\|=\sup _{\left\|x_{1}\right\|_{X} \leq 1, \ldots,\left\|x_{m+n}\right\|_{X} \leq 1}\left\|A\left(x_{1}, \ldots, x_{m+n}\right)\right\|_{Y}
$$

is finite. Similarly, an $(m, n)$-polynomial $P: X \rightarrow Y$ is continuous if and only if the value

$$
\|P\|=\sup _{\|x\|_{X} \leq 1}\|P(x)\|_{Y}
$$

is finite. Formula (4) implies the following inequality

$$
\left\|A_{P}^{(s)}\right\| \leq \frac{(m+n)^{m+n}}{m ! n !}\|P\| .
$$

\subsection{Algebraic combinations}

A mapping $f: T \rightarrow \mathbb{K}$, where $T$ is an arbitrary set and $\mathbb{K}=\mathbb{R}$ or $\mathbb{C}$, is called an algebraic combination of mappings $f_{1}, \ldots, f_{m}: T \rightarrow \mathbb{K}$ over $\mathbb{K}$ if there exists a polynomial $Q: \mathbb{K}^{m} \rightarrow \mathbb{K}$ such that

$$
f(x)=Q\left(f_{1}(x), \ldots, f_{m}(x)\right)
$$

for every $x \in T$.

A set $\left\{f_{1}, \ldots, f_{m}\right\}$ of mappings $f_{1}, \ldots, f_{m}: T \rightarrow \mathbb{K}$ is called algebraically independent if

$$
Q\left(f_{1}(x), \ldots, f_{m}(x)\right)=0
$$


for every $x \in T$ if and only if the polynomial $Q$ is identically equal to zero. If a set of mappings $\left\{f_{1}, \ldots, f_{m}\right\}$ is algebraically independent and polynomials $Q_{1}, Q_{2}: \mathbb{K}^{m} \rightarrow \mathbb{K}$ are such that

$$
Q_{1}\left(f_{1}(x), \ldots, f_{m}(x)\right)=Q_{2}\left(f_{1}(x), \ldots, f_{m}(x)\right)
$$

for every $x \in T$, then the polynomial $Q_{1}$ is identically equal to the polynomial $Q_{2}$. Thus, every algebraic combination of elements of an algebraically independent set of mappings is unique. An infinite set of mappings is called algebraically independent if every its finite subset is algebraically independent. A subset $\mathcal{B}$ of some algebra of mappings $\mathcal{A}$ is called an algebraic basis of $\mathcal{A}$ if every element of $\mathcal{A}$ can be uniquely represented as an algebraic combination of some elements of $\mathcal{B}$. Evidently, every algebraic basis is algebraically independent.

\subsection{The space $\ell_{p}\left(\mathbb{K}^{n}\right)$}

Let $n \in \mathbb{N}, p \in[1,+\infty)$ and $\mathbb{K}=\mathbb{R}$ or $\mathbb{C}$. Let us denote $\ell_{p}\left(\mathbb{K}^{n}\right)$ the vector space of all sequences $x=\left(x_{1}, x_{2}, \ldots\right)$, where $x_{j}=\left(x_{j}^{(1)}, \ldots, x_{j}^{(n)}\right) \in \mathbb{K}^{n}$ for $j \in \mathbb{N}$, such that the series $\sum_{j=1}^{\infty} \sum_{s=1}^{n}\left|x_{j}^{(s)}\right|^{p}$ is convergent. The space $\ell_{p}\left(\mathbb{K}^{n}\right)$ with norm

$$
\|x\|_{\ell_{p}\left(\mathbb{K}^{n}\right)}=\left(\sum_{j=1}^{\infty} \sum_{s=1}^{n}\left|x_{j}^{(s)}\right|^{p}\right)^{1 / p}
$$

is a Banach space.

Definition 1. A function $f$, defined on $\ell_{p}\left(\mathbb{K}^{n}\right)$, is called $\mathcal{S}$-symmetric (or just symmetric when the context is clear) if $f(x \circ \sigma)=f(x)$ for every $x \in \ell_{p}\left(\mathbb{K}^{n}\right)$ and for every bijection $\sigma \in \mathcal{S}$, where $x \circ \sigma=\left(x_{\sigma(1)}, x_{\sigma(2)}, \ldots\right)$.

For a multi-index $k=\left(k_{1}, \ldots, k_{n}\right) \in \mathbb{Z}_{+}^{n}$, let $|k|=k_{1}+\ldots+k_{n}$. For every $k \in \mathbb{Z}_{+}^{n}$ such that $|k| \geq\lceil p\rceil$, where $\lceil p\rceil$ is a ceiling of $p$, let us define a mapping $H_{k}^{\left(\mathbb{K}^{n}\right)}: \ell_{p}\left(\mathbb{K}^{n}\right) \rightarrow \mathbb{K}$ by

$$
H_{k}^{\left(K^{n}\right)}(x)=\sum_{j=1}^{\infty} \prod_{\substack{s=1 \\ k_{s}>0}}^{n}\left(x_{j}^{(s)}\right)^{k_{s}} .
$$

Note that $H_{k}^{\left(\mathbb{K}^{n}\right)}$ is an $\mathcal{S}$-symmetric $|k|$-homogeneous polynomial. We will use following result, proven in [11].

Proposition 3 ([11], Proposition 2). For $p \in[1,+\infty)$ and for every $k \in \mathbb{Z}_{+}^{n}$ such that $|k| \geq\lceil p\rceil$, the polynomial $H_{k}^{\left(\mathbb{C}^{n}\right)}$ on $\ell_{p}\left(\mathbb{C}^{n}\right)$ is continuous and $\left\|H_{k}^{\left(\mathbb{C}^{n}\right)}\right\| \leq 1$.

Theorem 1. Polynomials $H_{k}^{\left(\mathbb{C}^{n}\right)}$, where $k \in \mathbb{Z}_{+}^{n}$ are such that $|k| \geq\lceil p\rceil$, form an algebraic basis of the algebra of all $\mathcal{S}$-symmetric continuous complex-valued polynomials on $\ell_{p}\left(\mathbb{C}^{n}\right)$.

Note that Proposition 3 implies that for $p \in[1,+\infty)$ and for every $k \in \mathbb{Z}_{+}^{n}$ such that $|k| \geq$ $\lceil p\rceil$, the polynomial $H_{k}^{\left(\mathbb{R}^{n}\right)}$ on $\ell_{p}\left(\mathbb{R}^{n}\right)$ is continuous and $\left\|H_{k}^{\left(\mathbb{R}^{n}\right)}\right\| \leq 1$. 


\section{THE AlgEBRAIC BASIS OF THE ALgEBRA OF ALL SYMMETRIC CONTINUOUS POLYNOMIALS ON $\ell_{p}\left(\mathbb{R}^{n}\right)$}

Let $n \in \mathbb{N}$ and $p \in[1,+\infty)$. For every continuous $m$-homogeneous polynomial $P$ on $\ell_{p}\left(\mathbb{R}^{n}\right)$, which is, in general, complex-valued (we need this assumption for the sake of the applicability of results of the current section in section 3 ), let us define an $m$-homogeneous polynomial $\widehat{P}: \ell_{p}\left(\mathbb{C}^{n}\right) \rightarrow \mathbb{C}$ in the following way. Let $A_{P}^{(s)}$ be the $m$-linear symmetric mapping associated with $P$. Let $A_{\widehat{P}}^{(s)}: \underbrace{\ell_{p}\left(\mathbb{C}^{n}\right) \times \ldots \times \ell_{p}\left(\mathbb{C}^{n}\right)}_{m} \rightarrow \mathbb{C}$ be defined by

$$
A_{\widehat{P}}^{(s)}\left(z_{1}, \ldots, z_{m}\right)=\sum_{j_{1}=0}^{1} \ldots \sum_{j_{m}=0}^{1} i^{j_{1}+\ldots+j_{m}} A_{P}^{(s)}\left(w_{j_{1}}\left(z_{1}\right), \ldots, w_{j_{m}}\left(z_{m}\right)\right),
$$

where operators $w_{0}, w_{1}: \ell_{p}\left(\mathbb{C}^{n}\right) \rightarrow \ell_{p}\left(\mathbb{R}^{n}\right)$ are defined by

$$
\begin{aligned}
& w_{0}(z)=\left(\left(\operatorname{Re} x_{1}^{(1)}, \ldots, \operatorname{Re} x_{1}^{(n)}\right),\left(\operatorname{Re} x_{2}^{(1)}, \ldots, \operatorname{Re} x_{2}^{(n)}\right), \ldots\right), \\
& w_{1}(z)=\left(\left(\operatorname{Im} x_{1}^{(1)}, \ldots, \operatorname{Im} x_{1}^{(n)}\right),\left(\operatorname{Im} x_{2}^{(1)}, \ldots, \operatorname{Im} x_{2}^{(n)}\right), \ldots\right)
\end{aligned}
$$

for every $z=\left(\left(x_{1}^{(1)}, \ldots, x_{1}^{(n)}\right),\left(x_{2}^{(1)}, \ldots, x_{2}^{(n)}\right), \ldots\right) \in \ell_{p}\left(\mathbb{C}^{n}\right)$. Note that operators $w_{1}$ and $w_{2}$ are linear, continuous and $\left\|w_{0}\right\|=\left\|w_{1}\right\|=1$. It can be checked that $A_{\widehat{P}}^{(s)}$ is an $m$-linear symmetric mapping. By the continuity of mappings $A_{p}^{(s)}, w_{0}$ and $w_{1}$, the mapping $A_{\widehat{P}}^{(s)}$ is continuous. By (7), taking into account $\left\|w_{0}\right\|=\left\|w_{1}\right\|=1$,

$$
\left\|A_{\widehat{P}}^{(s)}\right\| \leq 2^{m}\left\|A_{P}^{(s)}\right\|
$$

Let $\widehat{P}$ be the restriction of $A_{\widehat{P}}^{(s)}$ to the diagonal. Since the mapping $A_{P}^{(s)}$ is continuous and $m$ linear, it follows that the mapping $\widehat{P}$ is a continuous $m$-homogeneous polynomial. By (3), (7) and (8),

$$
\|\widehat{P}\| \leq\left\|A_{\widehat{P}}^{(s)}\right\| \leq 2^{m}\left\|A_{P}^{(s)}\right\| \leq \frac{(2 m)^{m}}{m !}\|P\| .
$$

It can be checked that for every $m_{1}$-homogeneous polynomial $P_{1}$ and for every $m_{2}$-homogeneous polynomial $P_{2}$, which acts from $\ell_{p}\left(\mathbb{R}^{n}\right)$ to $\mathbb{C}$, where $m_{1}, m_{2} \in \mathbb{N}$, we have $\widehat{P_{1} P_{2}}=\widehat{P_{1}} \widehat{P}_{2}$.

For every continuous polynomial $P: \ell_{p}\left(\mathbb{R}^{n}\right) \rightarrow \mathbb{C}$ of the form (2), let

$$
\widehat{P}=P_{0}+\widehat{P}_{1}+\ldots+\widehat{P}_{K}
$$

Proposition 4. Let $\Gamma$ be an arbitrary index set. For every $\gamma \in \Gamma$, let $P_{\gamma}: \ell_{p}\left(\mathbb{R}^{n}\right) \rightarrow \mathbb{C}$ be a continuous $m_{\gamma}$-homogeneous polynomial, where $m_{\gamma} \in \mathbb{N}$. Suppose the set of polynomials $\left\{\widehat{P}_{\gamma}: \gamma \in \Gamma\right\}$ is algebraically independent. Then the set of polynomials $\left\{P_{\gamma}: \gamma \in \Gamma\right\}$ is algebraically independent.

Proof. Let $\Gamma_{0}$ be an arbitrary finite nonempty subset of $\Gamma$. Let us show that the set of polynomials $\left\{P_{\gamma}: \gamma \in \Gamma_{0}\right\}$ is algebraically independent. Suppose

$$
\alpha_{0}+\sum_{\mu=1}^{\mu^{\prime}} \sum_{\substack{l: \Gamma_{0} \rightarrow \mathbb{Z}_{+} \\ \varkappa(l)=\mu}} \alpha_{l} \prod_{\substack{\gamma \in \Gamma_{0} \\ l(\gamma)>0}}\left(P_{\gamma}(x)\right)^{l(\gamma)}=0
$$


for every $x \in \ell_{p}\left(\mathbb{R}^{n}\right)$, where $\alpha_{0}, \alpha_{l} \in \mathbb{C}, \mu^{\prime} \in \mathbb{N}, \varkappa(l)=\sum_{\gamma \in \Gamma_{0}} l(\gamma) m_{\gamma}$. For $\mu \in\left\{1, \ldots, \mu^{\prime}\right\}$, let

$$
Q_{\mu}(x)=\sum_{\substack{l: \Gamma_{0} \rightarrow \mathbb{Z}_{+} \\ \varkappa(l)=\mu}} \alpha_{l} \prod_{\substack{\gamma \in \Gamma_{0} \\ l(\gamma)>0}}\left(P_{\gamma}(x)\right)^{l(\gamma)}
$$

for every $x \in \ell_{p}\left(\mathbb{R}^{n}\right)$. By Proposition 1 , taking into account (10), $\alpha_{0}=0$ and, for every $\mu \in$ $\left\{1, \ldots, \mu^{\prime}\right\}$, the polynomial $Q_{\mu}$ is identically equal to zero, i.e., $\left\|Q_{\mu}\right\|=0$. By (9), $\left\|\widehat{Q}_{\mu}\right\| \leq$ $\frac{(2 \mu)^{\mu}}{\mu !}\left\|Q_{\mu}\right\|$. Therefore $\left\|\widehat{Q}_{\mu}\right\|=0$. Consequently, $\widehat{Q}_{\mu}$ is identically equal to zero, i.e.,

$$
\sum_{\substack{l: \Gamma_{0} \rightarrow \mathbb{Z}_{+} \\ \varkappa(l)=\mu}} \alpha_{l} \prod_{\substack{\gamma \in \Gamma_{0} \\ l(\gamma)>0}}\left(\widehat{P}_{\gamma}(z)\right)^{l(\gamma)}=0
$$

for every $z \in \ell_{p}\left(\mathbb{C}^{n}\right)$. Since the set of polynomials $\left\{\widehat{P}_{\gamma}: \gamma \in \Gamma_{0}\right\}$ is algebraically independent, it follows that every coefficient $\alpha_{l}$ is equal to zero. Thus, the set of polynomials $\left\{P_{\gamma}: \gamma \in \Gamma_{0}\right\}$ is algebraically independent.

Since every finite nonempty subset of the set of polynomials $\left\{P_{\gamma}: \gamma \in \Gamma\right\}$ is algebraically independent, it follows that the set $\left\{P_{\gamma}: \gamma \in \Gamma\right\}$ is algebraically independent.

Theorem 2. Let $P: \ell_{p}\left(\mathbb{R}^{n}\right) \rightarrow \mathbb{C}$ be a continuous m-homogeneous $\mathcal{S}$-symmetric polynomial. Then, in the case $1 \leq m<\lceil p\rceil$, the polynomial $P$ is identically equal to zero. In the case $m \geq\lceil p\rceil$, the polynomial $P$ can be uniquely represented in the form

$$
P(x)=\sum_{\substack{l: \Gamma_{m} \rightarrow \mathbb{Z}_{+} \\ \varkappa(l)=m}} \alpha_{l} \prod_{\substack{k \in \Gamma_{m} \\ l(k)>0}}\left(H_{k}^{\left(\mathbb{R}^{n}\right)}(x)\right)^{l(k)},
$$

where $x \in \ell_{p}\left(\mathbb{R}^{n}\right), \alpha_{l} \in \mathbb{C}, \Gamma_{m}=\left\{k \in \mathbb{Z}_{+}^{n}:\lceil p\rceil \leq|k| \leq m\right\}$ and $\varkappa(l)=\sum_{k \in \Gamma_{m}}|k| l(k)$.

Proof. Let $P$ be a continuous $m$-homogeneous $\mathcal{S}$-symmetric complex-valued polynomial on $\ell_{p}\left(\mathbb{R}^{n}\right)$, where $m \in \mathbb{N}$. Then $\widehat{P}$ is a continuous $m$-homogeneous complex-valued polynomial on $\ell_{p}\left(\mathbb{C}^{n}\right)$. Let us show that the polynomial $\widehat{P}$ is $\mathcal{S}$-symmetric. Let $z \in \ell_{p}\left(\mathbb{C}^{n}\right)$ and $\sigma \in \mathcal{S}$. Let us show that $\widehat{P}(z \circ \sigma)=\widehat{P}(z)$. By (1), taking into account that $P$ is $\mathcal{S}$-symmetric,

$$
\begin{aligned}
A_{P}^{(s)}\left(x_{1} \circ \sigma, \ldots, x_{m} \circ \sigma\right) & =\frac{1}{2^{m} m !} \sum_{\varepsilon_{1}, \ldots, \varepsilon_{m}= \pm 1} \varepsilon_{1} \ldots \varepsilon_{m} P\left(\varepsilon_{1} x_{1} \circ \sigma+\ldots+\varepsilon_{m} x_{m} \circ \sigma\right) \\
& =\frac{1}{2^{m} m !} \sum_{\varepsilon_{1}, \ldots, \varepsilon_{m}= \pm 1} \varepsilon_{1} \ldots \varepsilon_{m} P\left(\left(\varepsilon_{1} x_{1}+\ldots+\varepsilon_{m} x_{m}\right) \circ \sigma\right) \\
& =\frac{1}{2^{m} m !} \sum_{\varepsilon_{1}, \ldots, \varepsilon_{m}= \pm 1} \varepsilon_{1} \ldots \varepsilon_{m} P\left(\varepsilon_{1} x_{1}+\ldots+\varepsilon_{m} x_{m}\right)=A_{P}^{(s)}\left(x_{1}, \ldots, x_{m}\right)
\end{aligned}
$$

for every $x_{1}, \ldots, x_{m} \in \ell_{p}\left(\mathbb{R}^{n}\right)$. By (7) and (11), taking into account the equalities $w_{0}(z \circ \sigma)=$ $w_{0}(z)$ and $w_{1}(z \circ \sigma)=w_{1}(z)$,

$$
\begin{aligned}
\widehat{P}(z \circ \sigma)=A_{\widehat{P}}(\underbrace{z \circ \sigma, \ldots, z \circ \sigma}_{m}) & =\sum_{j_{1}=0}^{1} \ldots \sum_{j_{m}=0}^{1} i^{j_{1}+\ldots+j_{m}} A_{P}\left(w_{j_{1}}(z \circ \sigma), \ldots, w_{j_{m}}(z \circ \sigma)\right) \\
& =\sum_{j_{1}=0}^{1} \ldots \sum_{j_{m}=0}^{1} i^{j_{1}+\ldots+j_{m}} A_{P}\left(w_{j_{1}}(z) \circ \sigma, \ldots, w_{j_{m}}(z) \circ \sigma\right) \\
& =\sum_{j_{1}=0}^{1} \ldots \sum_{j_{m}=0}^{1} i^{j_{1}+\ldots+j_{m}} A_{P}\left(w_{j_{1}}(z), \ldots, w_{j_{m}}(z)\right)=\widehat{P}(z) .
\end{aligned}
$$


Thus, $\widehat{P}$ is $\mathcal{S}$-symmetric. So, $\widehat{P}$ is an $\mathcal{S}$-symmetric continuous $m$-homogeneous complex-valued polynomial on $\ell_{p}\left(\mathbb{C}^{n}\right)$.

Therefore, by Theorem 1 , the polynomial $\widehat{P}$ can be uniquely represented as an algebraic combination of polynomials $H_{k}^{\left(\mathbb{C}^{n}\right)}$, where $k \in \mathbb{Z}_{+}^{n}$ are such that $|k| \geq\lceil p\rceil$. Since every $H_{k}^{\left(\mathbb{C}^{n}\right)}$ is a $|k|$-homogeneous polynomial and $|k| \geq\lceil p\rceil$, it follows that, in the case $m<\lceil p\rceil$, the polynomial $\widehat{P}$ is identically equal to zero. In the case $m \geq\lceil p\rceil$, the polynomial $\widehat{P}$ is an algebraic combination of polynomials $H_{k}^{\left(\mathbb{C}^{n}\right)}$, where $k \in \mathbb{Z}_{+}^{n}$ are such that $m \geq|k| \geq\lceil p\rceil$, i.e.,

$$
\widehat{P}(z)=\sum_{\substack{l: \Gamma_{m} \rightarrow \mathbb{Z}_{+} \\ \varkappa(l)=m}} \alpha_{l} \prod_{\substack{k \in \Gamma_{m} \\ l(k)>0}}\left(H_{k}^{\left(\mathbb{C}^{n}\right)}(z)\right)^{l(k)},
$$

for every $z \in \ell_{p}\left(\mathbb{C}^{n}\right)$, where $\alpha_{l} \in \mathbb{C}, \Gamma_{m}=\left\{k \in \mathbb{Z}_{+}^{n}:\lceil p\rceil \leq|k| \leq m\right\}$ and $\varkappa(l)=\sum_{k \in \Gamma_{m}}|k| l(k)$. Since polynomials $P$ and $H_{k}^{\left(\mathbb{R}^{n}\right)}$ are restrictions to the space $\ell_{p}\left(\mathbb{R}^{n}\right)$ of polynomials $\widehat{P}$ and $H_{k}^{\left(\mathbb{C}^{n}\right)}$ respectively, by (12),

$$
P(x)=\sum_{\substack{l: \Gamma_{m} \rightarrow \mathbb{Z}_{+} \\ \varkappa(l)=m}} \alpha_{l} \prod_{\substack{k \in \Gamma_{m} \\ l(k)>0}}\left(H_{k}^{\left(\mathbb{R}^{n}\right)}(x)\right)^{l(k)}
$$

for every $x \in \ell_{p}\left(\mathbb{R}^{n}\right)$. By Theorem 1 , the set of polynomials $\left\{H_{k}^{\left(\mathbb{C}^{n}\right)}: k \in \Gamma_{m}\right\}$ is algebraically independent. Consequently, by Proposition 4, taking into account the equality $\widehat{H}_{k}^{\left(\mathbb{R}^{n}\right)}=H_{k}^{\left(\mathbb{C}^{n}\right)}$, the set of polynomials $\left\{H_{k}^{\left(\mathbb{R}^{n}\right)}: k \in \Gamma_{m}\right\}$ is algebraically independent over $\mathbb{C}$. Therefore, the representation (13) is unique.

Theorem 3. Polynomials $H_{k}^{\left(\mathbb{R}^{n}\right)}$, where $k \in \mathbb{Z}_{+}^{n}$ are such that $|k| \geq\lceil p\rceil$, form an algebraic basis of the algebra of all $\mathcal{S}$-symmetric continuous real-valued polynomials on $\ell_{p}\left(\mathbb{R}^{n}\right)$.

Proof. Let $P$ be a continuous $\mathcal{S}$-symmetric real-valued polynomial on $\ell_{p}\left(\mathbb{R}^{n}\right)$ of the form (2). Let us show that $P$ can be uniquely represented as an algebraic combination of some elements of the set $\left\{H_{k}^{\left(\mathbb{R}^{n}\right)}: k \in \mathbb{Z}_{+}^{n},|k| \geq\lceil p\rceil\right\}$. By Proposition 1 , for every $j \in\{1, \ldots, \operatorname{deg} P\}$, the $j$-homogeneous polynomial $P_{j}$ is continuous, $\mathcal{S}$-symmetric and real-valued. Therefore, by Theorem 2 , if $1 \leq j<\lceil p\rceil$, then the polynomial $P_{j}$ is identically equal to zero, otherwise

$$
P_{j}(x)=\sum_{\substack{l: \Gamma_{j} \rightarrow \mathbb{Z}_{+} \\ \varkappa_{j}(l)=j}} \alpha_{l} \prod_{\substack{k \in \Gamma_{j} \\ l(k)>0}}\left(H_{k}^{\left(\mathbb{R}^{n}\right)}(x)\right)^{l(k)}
$$

for every $x \in \ell_{p}\left(\mathbb{R}^{n}\right)$, where $\alpha_{l} \in \mathbb{C}, \Gamma_{j}=\left\{k \in \mathbb{Z}_{+}^{n}:\lceil p\rceil \leq|k| \leq j\right\}$ and $\varkappa_{j}(l)=\sum_{k \in \Gamma_{j}}|k| l(k)$. Let us show that all the coefficients $\alpha_{l}$ are real. Since the polynomial $P_{j}$ is real-valued, it follows that $P_{j}(x)-\overline{P_{j}(x)}=0$ for every $x \in \ell_{p}\left(\mathbb{R}^{n}\right)$, i.e.,

$$
2 i \sum_{\substack{l: \Gamma_{j} \rightarrow \mathbb{Z}_{+} \\ \varkappa_{j}(l)=j}} \operatorname{Im} \alpha_{l} \prod_{\substack{k \in \Gamma_{j} \\ l(k)>0}}\left(H_{k}^{\left(\mathbb{R}^{n}\right)}(x)\right)^{l(k)}=0
$$

for every $x \in \ell_{p}\left(\mathbb{R}^{n}\right)$. By Proposition 4 , the set of polynomials

$$
\left\{H_{k}^{\left(\mathbb{R}^{n}\right)}: k \in \mathbb{Z}_{+}^{n},|k| \geq\lceil p\rceil\right\}
$$


is algebraically independent over $\mathbb{C}$, therefore it is algebraically independent over $\mathbb{R}$. Consequently, by (14), $\operatorname{Im} \alpha_{l}=0$ for every coefficient $\alpha_{l}$, i.e., every $\alpha_{l}$ is real. Thus, we have that for every $x \in \ell_{p}\left(\mathbb{R}^{n}\right), P(x)=P_{0}$ in the case $\operatorname{deg} P<\lceil p\rceil$, and

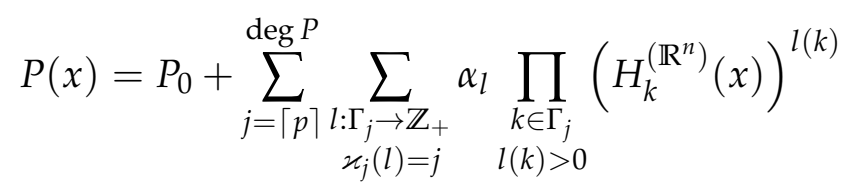

otherwise. Since the set of polynomials $\left\{H_{k}^{\left(\mathbb{R}^{n}\right)}: k \in \mathbb{Z}_{+}^{n},|k| \geq\lceil p\rceil\right\}$ is algebraically independent over $\mathbb{R}$, it follows that the representation (15) is unique.

\section{SYMMETRIC $*$-POLYNOMIALS ON $\ell_{p}\left(\mathbb{C}^{n}\right)$}

Let $n \in \mathbb{N}$ and $p \in[1,+\infty)$. Let the mapping $J: \ell_{p}\left(\mathbb{C}^{n}\right) \rightarrow \ell_{p}\left(\mathbb{R}^{2 n}\right)$ be defined by

$$
J(z)=\left(\left(\operatorname{Re} z_{1}^{(1)}, \operatorname{Im} z_{1}^{(1)}, \ldots, \operatorname{Re} z_{1}^{(n)}, \operatorname{Im} z_{1}^{(n)}\right),\left(\operatorname{Re} z_{2}^{(1)}, \operatorname{Im} z_{2}^{(1)}, \ldots, \operatorname{Re} z_{2}^{(n)}, \operatorname{Im} z_{2}^{(n)}\right), \ldots\right),
$$

where $z=\left(\left(z_{1}^{(1)}, \ldots, z_{1}^{(n)}\right),\left(z_{2}^{(1)}, \ldots, z_{2}^{(n)}\right), \ldots\right) \in \ell_{p}\left(\mathbb{C}^{n}\right)$. Let us show that the mapping $J$ is well-defined and bijective. Since all norms on $\mathbb{R}^{2}$ are equivalent, it follows that there exist constants $C>0$ and $c>0$ such that

$$
c \sqrt{\left|t_{1}\right|^{2}+\left|t_{2}\right|^{2}} \leq\left(\left|t_{1}\right|^{p}+\left|t_{2}\right|^{p}\right)^{1 / p} \leq C \sqrt{\left|t_{1}\right|^{2}+\left|t_{2}\right|^{2}}
$$

for every $\left(t_{1}, t_{2}\right) \in \mathbb{R}^{2}$. Therefore

$$
\begin{aligned}
\sum_{j=1}^{\infty} \sum_{s=1}^{n}\left(\left|\operatorname{Re} z_{j}^{(s)}\right|^{p}+\left|\operatorname{Im} z_{j}^{(s)}\right|^{p}\right) & \leq C^{p} \sum_{j=1}^{\infty} \sum_{s=1}^{n}\left(\sqrt{\left|\operatorname{Re} z_{j}^{(s)}\right|^{2}+\left|\operatorname{Im} z_{j}^{(s)}\right|^{2}}\right)^{p} \\
& =C^{p} \sum_{j=1}^{\infty} \sum_{s=1}^{n}\left|z_{j}^{(s)}\right|^{p}=C^{p}\|z\|_{\ell_{p}\left(\mathbb{C}^{n}\right)}^{p} .
\end{aligned}
$$

Thus, for every $z \in \ell_{p}\left(\mathbb{C}^{n}\right)$ the sequence $J(z)$ belongs to the space $\ell_{p}\left(\mathbb{R}^{2 n}\right)$ and $\|J(z)\|_{\ell_{p}\left(\mathbb{R}^{2 n}\right)}^{p} \leq$ $C^{p}\|z\|_{\ell_{p}\left(\mathbb{C}^{n}\right)}^{p}$, i.e.,

$$
\|J(z)\|_{\ell_{p}\left(\mathbb{R}^{2 n}\right)} \leq C\|z\|_{\ell_{p}\left(\mathbb{C}^{n}\right)} .
$$

Thus, the mapping $J$ is well-defined. Evidently, $J$ is injective. Let us show that $J$ is surjective. Let $x=\left(\left(x_{1}^{(1)}, x_{1}^{(2)}, \ldots, x_{1}^{(2 n-1)}, x_{1}^{(2 n)}\right),\left(x_{2}^{(1)}, x_{2}^{(2)}, \ldots, x_{2}^{(2 n-1)}, x_{2}^{(2 n)}\right), \ldots\right) \in \ell_{p}\left(\mathbb{R}^{2 n}\right)$. Let us construct $z_{x} \in \ell_{p}\left(\mathbb{C}^{n}\right)$ such that $J\left(z_{x}\right)=x$. Let $z_{x}=\left(\left(x_{1}^{(1)}+i x_{1}^{(2)}, \ldots, x_{1}^{(2 n-1)}+i x_{1}^{(2 n)}\right)\right.$, $\left.\left(x_{2}^{(1)}+i x_{2}^{(2)}, \ldots, x_{2}^{(2 n-1)}+i x_{2}^{(2 n)}\right), \ldots\right)$. Let us show that $z_{x}$ belongs to $\ell_{p}\left(\mathbb{C}^{n}\right)$. By (16),

$$
\begin{aligned}
\sum_{j=1}^{\infty} \sum_{s=1}^{n}\left|x_{j}^{(2 s-1)}+i x_{j}^{(2 s)}\right|^{p} & =\sum_{j=1}^{\infty} \sum_{s=1}^{n}\left(\sqrt{\left|x_{j}^{(2 s-1)}\right|^{2}+\left|x_{j}^{(2 s)}\right|^{2}}\right)^{p} \\
& \leq \sum_{j=1}^{\infty} \sum_{s=1}^{n}\left(\frac{1}{c}\left(\left|x_{j}^{(2 s-1)}\right|^{p}+\left|x_{j}^{(2 s)}\right|^{p}\right)^{1 / p}\right)^{p} \\
& =\frac{1}{c^{p}} \sum_{j=1}^{\infty} \sum_{s=1}^{n}\left(\left|x_{j}^{(2 s-1)}\right|^{p}+\left|x_{j}^{(2 s)}\right|^{p}\right)=\frac{1}{c^{p}}\|x\|_{\ell_{p}\left(\mathbb{R}^{2 n}\right)}^{p} .
\end{aligned}
$$


Thus, $z_{x}$ belongs to the space $\ell_{p}\left(\mathbb{C}^{n}\right)$ and $\left\|z_{x}\right\|_{\ell_{p}\left(\mathbb{C}^{n}\right)}^{p} \leq \frac{1}{c^{p}}\|x\|_{\ell_{p}\left(\mathbb{R}^{2 n}\right)}^{p}$, i.e., taking into account the equality $J\left(z_{x}\right)=x$,

$$
\left\|J^{-1}(x)\right\|_{\ell_{p}\left(\mathbb{C}^{n}\right)} \leq \frac{1}{c}\|x\|_{\ell_{p}\left(\mathbb{R}^{2 n}\right)}
$$

for every $x \in \ell_{p}\left(\mathbb{R}^{2 n}\right)$. Hence, the mapping $J$ is bijective. Note that the mapping $J$ is real-linear, i.e., it is additive and $J(\lambda z)=\lambda J(z)$ for every $\lambda \in \mathbb{R}$ and $z \in \ell_{p}\left(\mathbb{C}^{n}\right)$. By (17) and (18), both mappings $J$ and $J^{-1}$ are continuous.

Proposition 5. For every continuous $\mathcal{S}$-symmetric $\left(m_{1}, m_{2}\right)$-polynomial $P: \ell_{p}\left(\mathbb{C}^{n}\right) \rightarrow \mathbb{C}$ the mapping $P \circ J^{-1}$ is a continuous $\mathcal{S}$-symmetric $\left(m_{1}+m_{2}\right)$-homogeneous polynomial, acting from $\ell_{p}\left(\mathbb{R}^{2 n}\right)$ to $\mathbb{C}$.

Proof. Let $P: \ell_{p}\left(\mathbb{C}^{n}\right) \rightarrow \mathbb{C}$ be a continuous $\mathcal{S}$-symmetric $\left(m_{1}, m_{2}\right)$-polynomial. Let $A_{P}^{(s)}$ be the $\left(m_{1}, m_{2}\right)$-symmetric $\left(m_{1}, m_{2}\right)$-linear mapping, associated with $P$. Let the mapping $B_{\widetilde{P}}:\left(\ell_{p}\left(\mathbb{R}^{2 n}\right)\right)^{m_{1}+m_{2}} \rightarrow \mathbb{C}$ be defined by

$$
B_{\widetilde{P}}\left(x_{1}, \ldots, x_{m_{1}+m_{2}}\right)=A_{P}\left(J^{-1}\left(x_{1}\right), \ldots, J^{-1}\left(x_{m_{1}+m_{2}}\right)\right),
$$

where $x_{1}, \ldots, x_{m_{1}+m_{2}} \in \ell_{p}\left(\mathbb{R}^{2 n}\right)$. Since $J^{-1}$ is real-linear and $A_{P}$ is $\left(m_{1}, m_{2}\right)$-linear, it follows that $B_{\widetilde{P}}$ is an $\left(m_{1}+m_{2}\right)$-linear mapping. By (6) and (18),

$$
\begin{aligned}
\left\|B_{\widetilde{P}}\right\| & =\sup _{\left\|x_{1}\right\|_{\ell_{p}\left(\mathbb{R}^{2 n}\right)} \leq 1, \ldots,\left\|x_{m_{1}+m_{2}}\right\|_{\ell_{p}\left(\mathbb{R}^{2 n}\right)} \leq 1}\left|B_{\widetilde{P}}\left(x_{1}, \ldots, x_{m_{1}+m_{2}}\right)\right| \\
& =\sup _{\left\|x_{1}\right\|_{\ell_{p}\left(\mathbb{R}^{2 n}\right)} \leq 1, \ldots,\left\|x_{m_{1}+m_{2}}\right\|_{\ell_{p}\left(\mathbb{R}^{2 n}\right)} \leq 1}\left|A_{P}\left(J^{-1}\left(x_{1}\right), \ldots, J^{-1}\left(x_{m_{1}+m_{2}}\right)\right)\right| \\
& \leq \sup _{\left\|x_{1}\right\|_{\ell_{p}\left(\mathbb{R}^{2 n}\right)} \leq 1, \ldots,\left\|x_{m_{1}+m_{2}}\right\|_{\ell_{p}\left(\mathbb{R}^{2 n}\right)} \leq 1}\left\|A_{P}\right\|\left\|J^{-1}\left(x_{1}\right)\right\|_{\ell_{p}\left(\mathbb{C}^{n}\right)} \ldots\left\|J^{-1}\left(x_{m_{1}+m_{2}}\right)\right\|_{\ell_{p}\left(\mathbb{C}^{n}\right)} \\
& \leq \frac{\left\|A_{P}\right\|}{c^{m_{1}+m_{2}}}\left\|x_{1}\right\|_{\ell_{p}\left(\mathbb{R}^{2 n}\right) \leq 1, \ldots,\left\|x_{m_{1}+m_{2}}\right\|_{\ell p\left(\mathbb{R}^{2 n}\right)} \leq 1}\left\|x_{1}\right\|_{\ell_{p}\left(\mathbb{R}^{2 n}\right)} \ldots\left\|x_{m_{1}+m_{2}}\right\|_{\ell_{p}\left(\mathbb{R}^{2 n}\right)} \\
& =\frac{\left\|A_{P}\right\|}{c^{m_{1}+m_{2}}} \leq \frac{\left(m_{1}+m_{2}\right)^{m_{1}+m_{2}}\|P\|}{m_{1} ! m_{2} ! c^{m_{1}+m_{2}}} .
\end{aligned}
$$

Thus, $\left\|B_{\widetilde{P}}\right\|$ is finite and, consequently, $B_{\widetilde{P}}$ is continuous. Let $\widetilde{P}$ be the restriction to the diagonal of $B_{\widetilde{P}}$. Then $\widetilde{P}$ is the $\left(m_{1}+m_{2}\right)$-homogeneous polynomial. Since $\|\widetilde{P}\| \leq\left\|B_{\widetilde{P}}\right\|$, it follows that $\widetilde{P}$ is continuous. Note that $\widetilde{P}=P \circ J^{-1}$. Let us show that $\widetilde{P}$ is $\mathcal{S}$-symmetric. Let $x \in \ell_{p}\left(\mathbb{R}^{2 n}\right)$ and $\sigma \in \mathcal{S}$. Note that $J^{-1}(x \circ \sigma)=J^{-1}(x) \circ \sigma$. Therefore, since $P$ is $\mathcal{S}$-symmetric,

$$
\widetilde{P}(x \circ \sigma)=P\left(J^{-1}(x \circ \sigma)\right)=P\left(J^{-1}(x) \circ \sigma\right)=P\left(J^{-1}(x)\right)=\widetilde{P}(x) .
$$

Thus, $\widetilde{P}$ is $\mathcal{S}$-symmetric.

Theorem 4. The set of mappings $\left\{H_{k}^{\left(\mathbb{R}^{2 n}\right)} \circ J: k \in \mathbb{Z}_{+}^{2 n},|k| \geq\lceil p\rceil\right\}$ is an algebraic basis of the algebra of all continuous $\mathcal{S}$-symmetric $*$-polynomials, acting from $\ell_{p}\left(\mathbb{C}^{n}\right)$ to $\mathbb{C}$.

Proof. Let $P: \ell_{p}\left(\mathbb{C}^{n}\right) \rightarrow \mathbb{C}$ be a continuous $\mathcal{S}$-symmetric $*$-polynomial of the form (5). By Proposition 2, taking into account the continuity and the $\mathcal{S}$-symmetry of $P$, for every $t \in$ $\{0, \ldots, K\}$ and $j \in\{0, \ldots, t\}$, the $(j, t-j)$-polynomial $P_{j, t-j}$ is continuous and $\mathcal{S}$-symmetric. 
Therefore, by Proposition 5, the mapping $P_{j, t-j} \circ J^{-1}$ is a continuous $\mathcal{S}$-symmetric $t$-homogeneous polynomial, acting from $\ell_{p}\left(\mathbb{R}^{2 n}\right)$ to $\mathbb{C}$. Consequently, by Theorem 2 , the polynomial $P_{j, t-j} \circ J^{-1}$ is identically equal to zero in the case $1 \leq t<\lceil p\rceil$, and, otherwise, the polynomial $P_{j, t-j} \circ J^{-1}$ can be uniquely represented in the form

$$
\left(P_{j, t-j} \circ J^{-1}\right)(x)=\sum_{\substack{l: \Gamma_{t} \rightarrow \mathbb{Z}_{+} \\ \varkappa_{t}(l)=t}} \alpha_{l}^{(j, t-j)} \prod_{\substack{k \in \Gamma_{t} \\ l(k)>0}}\left(H_{k}^{\left(\mathbb{R}^{2 n}\right)}(x)\right)^{l(k)},
$$

where $x \in \ell_{p}\left(\mathbb{R}^{2 n}\right), \alpha_{l}^{(j, t-j)} \in \mathbb{C}, \Gamma_{t}=\left\{k \in \mathbb{Z}_{+}^{2 n}:\lceil p\rceil \leq|k| \leq t\right\}$ and $\varkappa_{t}(l)=\sum_{k \in \Gamma_{t}}|k| l(k)$. Therefore, taking into account that $J$ is a bijection, the mapping $P_{j, t-j}$ is identically equal to zero in the case $1 \leq t<\lceil p\rceil$, and

$$
P_{j, t-j}(z)=\sum_{\substack{l: \Gamma_{t} \rightarrow \mathbb{Z}_{+} \\ \varkappa_{t}(l)=t}} \alpha_{l}^{(j, t-j)} \prod_{\substack{k \in \Gamma_{t} \\ l(k)>0}}\left(\left(H_{k}^{\left(\mathbb{R}^{2 n}\right)} \circ J\right)(z)\right)^{l(k)},
$$

for every $z \in \ell_{p}\left(\mathbb{C}^{n}\right)$, otherwise. Consequently, $P=P_{0}$ in the case $\operatorname{deg} P<\lceil p\rceil$, and

$$
P(z)=P_{0}+\sum_{t=\lceil p\rceil}^{\operatorname{deg} P} \sum_{j=0}^{t} \sum_{\substack{l: \Gamma_{t} \rightarrow \mathbb{Z}_{+} \\ \varkappa_{t}(l)=t}} \alpha_{l}^{(j, t-j)} \prod_{\substack{k \in \Gamma_{t} \\ l(k)>0}}\left(\left(H_{k}^{\left(\mathbb{R}^{2 n}\right)} \circ J\right)(z)\right)^{l(k)}
$$

for every $z \in \ell_{p}\left(\mathbb{C}^{n}\right)$, otherwise. By Proposition 4 , the set of polynomials $\left\{H_{k}^{\left(\mathbb{R}^{2 n}\right)}: k \in\right.$ $\left.\mathbb{Z}_{+}^{2 n},|k| \geq\lceil p\rceil\right\}$ is algebraically independent. Since $J$ is a bijection, it follows that the set of *-polynomials $\left\{H_{k}^{\left(\mathbb{R}^{2 n}\right)} \circ J: k \in \mathbb{Z}_{+}^{2 n},|k| \geq\lceil p\rceil\right\}$ is algebraically independent. Therefore, the representation (19) is unique.

\section{REFERENCES}

[1] Alencar R., Aron R., Galindo P., Zagorodnyuk A. Algebras of symmetric holomorphic functions on $\ell_{p}$. Bull. Lond. Math. Soc. 2003, 35, 55-64. doi:10.1112/S0024609302001431

[2] Aron R., Falcó J., Maestre M. Separation theorems for group invariant polynomials. J. Geom. Anal. 2018, 28 (1), 393-404. doi:10.1007/s12220-017-9825-0

[3] Aron R., Galindo P., Pinasco D., Zalduendo I. Group-symmetric holomorphic functions on a Banach space. Bull. Lond. Math. Soc. 2016, 48 (5), 779--796. doi:10.1112/blms/bdw043

[4] Chernega I., Galindo P., Zagorodnyuk A. Some algebras of symmetric analytic functions and their spectra. Proc. Edinburgh Math. Soc. 2012, 55 (1), 125-142. doi:10.1017/S0013091509001655

[5] Galindo P., Vasylyshyn T., Zagorodnyuk A. The algebra of symmetric analytic functions on $L_{\infty}$. Proc. Roy. Soc. Edinburgh Sect. A 2017, 147 (4),743-761. doi:10.1017/S0308210516000287

[6] Galindo P., Vasylyshyn T., Zagorodnyuk A. Symmetric and finitely symmetric polynomials on the spaces $\ell_{\infty}$ and $L_{\infty}[0,+\infty)$. Math. Nachr. 2018, 291 (11-12), 1712-1726. doi:10.1002/mana.201700314

[7] Galindo P., Vasylyshyn T., Zagorodnyuk A. Analytic structure on the spectrum of the algebra of symmetric analytic functions on $L_{\infty}$. Rev. R. Acad. Cienc. Exactas Fís. Nat. Ser. A Math. RACSAM2020, 114 (56). doi:10.1007/s13398-020-00791-w

[8] González M., Gonzalo R., Jaramillo J. A. Symmetric polynomials on rearrangement invariant function spaces. J. Lond. Math. Soc. 1999, 59 (2), 681-697. doi:10.1112/S0024610799007164 
[9] Halushchak S. Spectra of some algebras of entire functions of bounded type, generated by a sequence of polynomials. Carpathian Math. Publ. 2019, 11 (2), 311-320. doi:10.15330/cmp.11.2.311-320

[10] Kravtsiv V. Algebraic basis of the algebra of block-symmetric polynomials on $\ell_{1} \oplus \ell_{\infty}$. Carpathian Math. Publ. 2019, 11 (1), 89-95. doi:10.15330/cmp.11.1.89-95

[11] Kravtsiv V., Vasylyshyn T., Zagorodnyuk A. On algebraic basis of the algebra of symmetric polynomials on $\ell_{p}\left(\mathbb{C}^{n}\right)$. J. Funct. Spaces 2017, 2017. doi:10.1155/2017/4947925

[12] Martin R. S. Contribution to the theory of functionals. Ph.D. thesis, University of California, 1932.

[13] Mitrofanov M. A. Approximation of continuous functions on complex Banach spaces. Math. Notes 2009, 86 (3-4), 530-541. doi:10.1134/S0001434609090302

[14] Mujica J. Complex Analysis in Banach Spaces. North Holland, 1986.

[15] Nemirovskii A. S., Semenov S. M. On polynomial approximation of functions on Hilbert space. Math. USSR Sb. 1973, 21 (2), 255-277. doi:10.1070/SM1973v021n02ABEH002016

[16] Vasylyshyn T.V. Symmetric *-polynomials on $\mathbb{C}^{n}$. Carpathian Math. Publ. 2018, 10 (2), 395-401. doi: 10.15330/cmp.10.2.395-401

[17] Vasylyshyn T. Point-evaluation functionals on algebras of symmetric functions on $\left(L_{\infty}\right)^{2}$. Carpathian Math. Publ. 2019, 11 (2), 493-501. doi:10.15330/cmp.11.2.493-501

[18] Vasylyshyn T. Symmetric polynomials on $\left(L_{p}\right)^{n}$. Eur. J. Math. 2020, 6 (1), 164-178. doi:10.1007/s40879-018-0268-3

[19] Vasylyshyn T. V. Symmetric polynomials on the Cartesian power of $L_{p}$ on the semi-axis. Mat. Stud. 2018, 50 (1), 93-104. doi:10.15330/ms.50.1.93-104

[20] Vasylyshyn T.V. The algebra of symmetric polynomials on $\left(L_{\infty}\right)^{n}$. Mat. Stud. 2019, 52 (1), 71-85. doi: 10.30970/ms.52.1.71-85

[21] Vasylyshyn T., Zagorodnyuk A. Continuous symmetric 3-homogeneous polynomials on spaces of Lebesgue measurable essentially bounded functions. Methods Funct. Anal. Topology 2018, 24 (4), 381-398.

[22] Vasylyshyn T. V., Zagorodnyuk A. V. Polarization formula for $(p, q)$-polynomials on a complex normed space. Methods Funct. Anal. Topology 2011, 17 (1), 75-83.

[23] Vasylyshyn T. Algebras of entire symmetric functions on spaces of Lebesgue-measurable essentially bounded functions. J. Math. Sci. 2020, 246, 264-276. doi:10.1007/s10958-020-04736-x

Received 25.03.2020

Василишин Т.В. Симетричні функиії на просторах $\ell_{p}\left(\mathbb{R}^{n}\right) i \ell_{p}\left(\mathbb{C}^{n}\right) / /$ Карпатські матем. публ. - 2020. - T.12, №1. - C. 5-16.

$\triangle$ ана робота присвячена вивченню алгебр неперервних симетричних, тобто, інваріантних відносно перестановок координат їхніх аргументів, поліномів і $*$-поліномів на банахових просторах $\ell_{p}\left(\mathbb{R}^{n}\right)$ і $\ell_{p}\left(\mathbb{C}^{n}\right)$ всіх сумовних у степені $p$ послідовностей $n$-вимірних векторів дійсних і комплексних чисел відповідно, де $1 \leq p<+\infty$.

Сконструйовано підмножину алгебри всіх неперервних симетричних поліномів на простоpi $\ell_{p}\left(\mathbb{R}^{n}\right)$ таку, шо кожен неперервний симетричний поліном на просторі $\ell_{p}\left(\mathbb{R}^{n}\right)$ може бути єАиним чином поданий у вигляді лінійної комбінації добутків елементів цієї множини. Іншими словами, сконструйовано алгебраїчний базис алгебри всіх неперервних симетричних поліномів на просторі $\ell_{p}\left(\mathbb{R}^{n}\right)$. Використовуючи даний результат, сконструйовано алгебраїчний базис алгебри всіх неперервних симетричних $*$-поліномів на просторі $\ell_{p}\left(\mathbb{C}^{n}\right)$.

Результати даної роботи можуть бути використані для досліджень алгебр, згенерованих неперервними симетричними поліномами на просторі $\ell_{p}\left(\mathbb{R}^{n}\right)$, і алгебр, згенерованих неперервними симетричними $*$-поліномами на просторі $\ell_{p}\left(\mathbb{C}^{n}\right)$.

Ключові слова і фрази: поліном, *-поліном, симетричний поліном, симетричний $*$-поліном, алгебраїчний базис. 